Check for updates

Cite this: RSC Adv., 2017, 7, 34331

\title{
Microfibrous silver-coated polymeric scaffolds with tunable mechanical properties $\uparrow$
}

\author{
Parvathalu. Kalakonda, ${ }^{\text {ab }}$ Musab A. Aldhahri, ${ }^{a}$ Mohamed Shaaban Abdel-wahab, ${ }^{a}$ \\ Ali Tamayol, ${ }^{\text {cde }}$ K. Mollazadeh Moghaddam, ${ }^{\text {cd }}$ Fathia Ben Rached, ${ }^{\mathrm{b}}$ Arnab Pain, ${ }^{\mathrm{b}}$ \\ Ali Khademhosseini, (D) ${ }^{\text {cdefg }}$ Adnan Memic ${ }^{\star a}$ and Sahraoui Chaieb (D) t $^{* b h}$
}

Electrospun scaffolds of poly(glycerol sebacate)/poly( $\varepsilon$-caprolactone) (PGS/PCL) have been used for engineered tissues due to their desirable thermal and mechanical properties as well as their tunable degradability. In this paper, we fabricated micro-fibrous scaffolds from a composite of PGS/PCL using a standard electrospinning method and coated them with silver (Ag). The low temperature coating method prevented substrate melting and the Ag coating decreases the pore size and increases the diameter of fibers which resulted in enhanced thermal and mechanical properties. We further compared the mechanical properties of the composite fibrous scaffolds with different thicknesses of Ag coated scaffolds. The composite fibrous scaffold with a $275 \mathrm{~nm} \mathrm{Ag}$ coating showed higher tensile modulus $(E)$ and ultimate tensile strength (UTS) without any post-processing treatment. Lastly, potential controlled release of the Ag coating from the composite fibrous scaffolds could present interesting biomedical applications.

Received 12th October 2016 Accepted 14th June 2017

DOI: $10.1039 / c 6 r a 25151 j$

rsc.li/rsc-advances increases the chances of bacterial infection. ${ }^{6,7}$ Bacterial infection not only hinders wound healing and treatment, but also may ultimately lead to sepsis and death in severe cases. One promising strategy for wound management against bacterial infection is through the use of wound-dressings and materials with antibacterial activity.

Previously, many natural polymers such as gelatin, collagen, alginate, and their composites have been proposed for biomedical applications including development of wound healing scaffolds. A composite of gelatin containing silver nanoparticles was proposed as an efficient wound dressing material with antimicrobial activity. ${ }^{8}$ It was found that this composite gel has an antibacterial effect on a wide range of pathogens such as $S$. typhimurium, L. monocytogenes, E. coli, S. aureus, and $B$. cereus. These pathogens were significantly inhibited in a dose-dependent manner where the Gramnegative pathogens were more inhibited than the Grampositive. $\mathrm{TiO}_{2}, \mathrm{ZnO}, \mathrm{CuO}$, and $\mathrm{MgO}$ as well as carbon nanotubes were also used as additive compounds to wound dressing to inhibit bacterial activity but they were less efficient than silver nanoparticle- based composites. ${ }^{\mathbf{9 , 1 0}}$

Such scaffolds, albeit having a high degree of biocompatibility, are often plagued by a low shear modulus. They cannot withstand any tension or shearing without spreading due to their shear-thinning property (gelatin) ${ }^{\mathbf{1 1}}$ or low mechanical strength (collagen $^{\mathbf{1 2}}$ or alginate ${ }^{\mathbf{1 3}}$ ). Synthetic polymers however often offer higher mechanical properties than natural hydrogels. Scaffolds of such polymers fabricated using electrospinning also possess morphological features similar to native 
extracellular matrix, which has made them excellent candidates for tissue engineering applications. Previously, poly(glycerol sebacate) (PGS) has been shown to be a promising polymer in the development of scaffold materials for soft tissue engineering applications due to its unique blend of mechanical and biological properties. ${ }^{\mathbf{1 4 - 1 6}}$ The precursors of PGS (glycerol and sebacic acid) have also been approved by the FDA. ${ }^{\mathbf{1 6}, 17}$ PGS elastomeric polymer has attracted significant attention because of its easy synthesis, bio-degradability, ${ }^{18-20}$ biocompatibility, ${ }^{21}$ and has emerged as a promising material for tissue engineering application. ${ }^{14-17,22-26}$ Furthermore, recent studies have shown that PGS-based scaffolds have remarkable cellular responses in several biocompatibility tests. ${ }^{17,27}$

Previous reports have, however, shown that pure PGS polymer, especially at low molecular weights cannot be used in electrospinning process. The formation of PGS nanofibers could however be achieved by an addition of an electrospinnable polymer such as gelatin or poly(e-caprolactone) (PCL). ${ }^{28-31}$ PCL is an FDA-approved, semi-crystalline biodegradable hydrophobic polymer that has exhibited a poor cell attachment due to its hydrophobicity. ${ }^{27-32}$ Since PGS and PCL possess different physical properties, their blend offers interesting novel characteristics.

Scaffolds used in wound healing applications should have antibacterial activity to inhibit bacterial colonization. Thus, the fabrication of a fibrous scaffold with antibacterial coatings and superior mechanical properties is of great importance. Previously, coatings of antibacterial materials such as metal nanoparticles, ${ }^{33,34}$ medicaments, ${ }^{35-37}$ antibiotics ${ }^{38}$ on the composite of fibrous scaffold have been shown to have the potential to minimize the risk of microbial infections but their burst release and safety concerns, due to their possible toxicity, have hindered their use.

In the present study, we fabricated PGS/PCL composite scaffolds by using the electro-spinning method. The composite fibrous scaffolds were stable without any post processing, such as thermal annealing ${ }^{39-41}$ etc. When the fibrous scaffolds were made by PGS/PCL blending we observed an improvement in the mechanical properties of scaffolds when compared to other combinations. ${ }^{\mathbf{1 7}, \mathbf{4 2}} \mathrm{Next}$, a thin layer of $\mathrm{Ag}$ with tunable thicknesses was coated on the fibers. The effects of Ag coating on the thermal, mechanical, compositional, and antibacterial activity of the fibrous scaffolds were measured. Ultimately, an optimal $\mathrm{Ag}$ coating layer enhanced significantly the mechanical, and viscoelastic properties as well as its thermal stability. It can also act as a desirable antibacterial agent for possible clinical applications.

\section{Results}

In this study, we used the electrospinning method to fabricate fibrous scaffolds of PGS/PCL with a ratio $1: 1$ that was then coated with different thickness of silver (Ag) in a controlled manner by using a RF sputtering method (Fig. S1†). The electrospinning method is excellent to develop composite scaffolds and does not require any post-processing. In this study, we mainly focused on thermal and mechanical properties of Agcoated composite scaffold of PGS/PCL.

To probe the chemical compositions of the composite scaffolds, we used FTIR analysis (Fig. 1). All of the functional bands of PGS are overlapped with PCL bands. There were two primary band detected at $1723 \mathrm{~cm}^{-1}$ stretching vibration of carboxyl $(\mathrm{C}=\mathrm{O})$ and $1167 \mathrm{~cm}^{-1}$ stretching vibration of an ether group (C-O) in the spectrum. ${ }^{24}$ There were other stretching bands observed such as symmetric C-H stretching at $2926 \mathrm{~cm}^{-1}, \mathrm{C}-\mathrm{O}$, and $\mathrm{C}-\mathrm{C}$ stretching vibrations at $1295 \mathrm{~cm}^{-1}$. Stretching bands of methylene groups ${ }^{43}$ can be seen at 1360, 2930, $2860 \mathrm{~cm}^{-1}$ and the ester band $\mathrm{C}-\mathrm{O}$ vibration at 1166, 1723, 930 and $1287 \mathrm{~cm}^{-1}$. This indicated the formation of composite nanofibers made up of both PGS and PCL polymers. Fig. S $2 \uparrow$ shows the various FTIR of the polymers and their blends as well as the blend sputtered with Ag.

The surface morphology of fibrous composite scaffolds with different Ag coatings is shown in Fig. 2A. The diameter and pore size distribution of pristine scaffold were measured using an SEM. The average diameter of electrospun fibers and pore size of the pristine scaffold, which were found to be approximately $400 \mathrm{~nm}$ and $600 \mathrm{~nm}$ respectively, were measured by statistical analysis of a normal distribution based on SEM images (Fig. 2Bi and ii).

The mechanical characterization was carried out on the fibrous scaffold with different Ag surface coatings. We varied the $\mathrm{Ag}$ coating from $50 \pm 6$ to $275 \pm 6 \mathrm{~nm}$ (Fig. S1 $\dagger$ ). This process is easily reproducible by controlling the RF sputtering parameters such as deposition time, gas pressure and power intensity. The fibrous scaffold used for all mechanical measurements were rectangular in shape with an average thickness of 100-200 $\mu \mathrm{m}$. We did not use dogbone-shaped samples because their width to length ratio are large and the stress is fairly uniform along these samples. Fig. 3A shows the linear region of the stress-strain curve for all scaffolds in the first 10-15\% strain range. The mechanical properties of scaffolds particularly depend on several microstructure parameters such as the fiber diameter, the pore diameter, the outer metal coating, the ratio and spatial distribution of constituent fibers in the scaffold. The

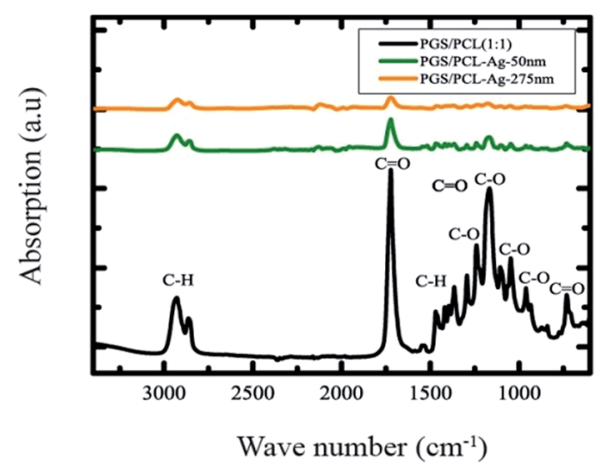

Fig. 1 FTIR spectra of the fibrous scaffold of PGS/PCL compared to Ag coating scaffold. Individual polymers were as previously reported in literature. ${ }^{44}$ Fig. S2 in the ESI† shows the spectra of the individual polymers. 


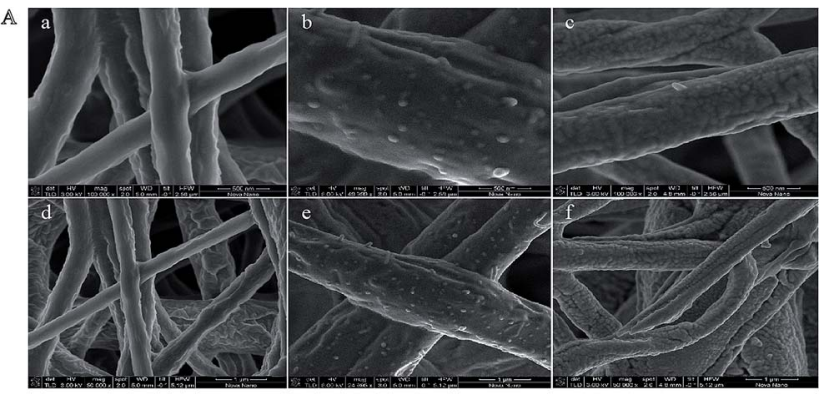

$\mathbb{B}$
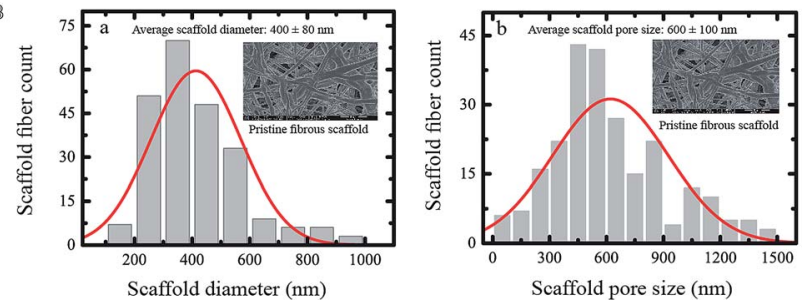

Fig. 2 (A) Representative SEM images of the pristine fibrous PGS/PCL scaffold and various Ag-coated fibrous scaffolds (i), PGS/PCL; (ii) $50 \mathrm{~nm}$, and (iii) $275 \mathrm{~nm}$ Ag-coated fibrous scaffold. (B) Pristine fibrous scaffold distribution of diameter (i) and pore size (ii).

distribution of diameters and pore sizes for fibrous Ag-coated scaffolds is shown in Fig. 2B. The tensile modulus of uncoated PGS/PCL $(1: 1)$ scaffolds was found to be around 3.3 MPa. The mechanical properties of the Ag-coated scaffolds were increased several fold. The tensile modulus of the fibrous scaffold coated with a $275 \mathrm{~nm} \mathrm{Ag}$ film increased by $250 \%$ (Fig. 3B). The ultimate tensile strength of fibrous scaffold increased monotonously as a function of $\mathrm{Ag}$ coating (Fig. 4A).

The ultimate tensile strength of the scaffold with $275 \mathrm{~nm} \mathrm{Ag}$ film was approximately $1.8(180 \%)$ times that of the pristine scaffold. Interestingly, the tensile strain (elongation) increased about $300 \%$ at $50 \mathrm{~nm} \mathrm{Ag-coated} \mathrm{scaffold} \mathrm{and} \mathrm{then} \mathrm{decreased} \mathrm{to}$ about $\mathbf{1 7 5 \%}$ (lower than the pristine scaffold) for higher $\mathrm{Ag}$ coating (Fig. 4B). The higher Ag coating may lead to more brittle scaffold due to the stronger interfacial interaction between $\mathrm{Ag}$ and the scaffold fibers. The fibrous scaffold with $275 \mathrm{~nm}$ coating showed higher modulus $(E)$, higher UTS, and higher stiffness
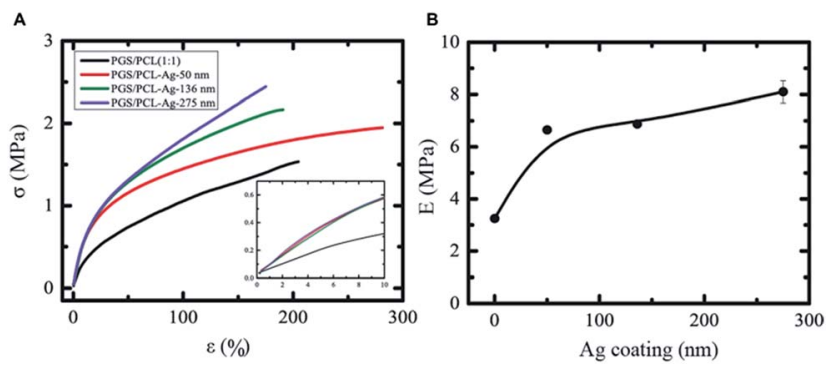

Fig. 3 Mechanical properties of the fibrous scaffold of PGS/PCL. (A) The tensile stress $(\sigma)$ versus tensile strain $(E)$ curves of the fibrous scaffold (PCL/PGS-1 : 1) and Ag-coated fibrous scaffold. (B) The tensile modulus $(E)$ of the $\mathrm{Ag}$-coated scaffolds increased by about $250 \%$ with the Ag-coating.

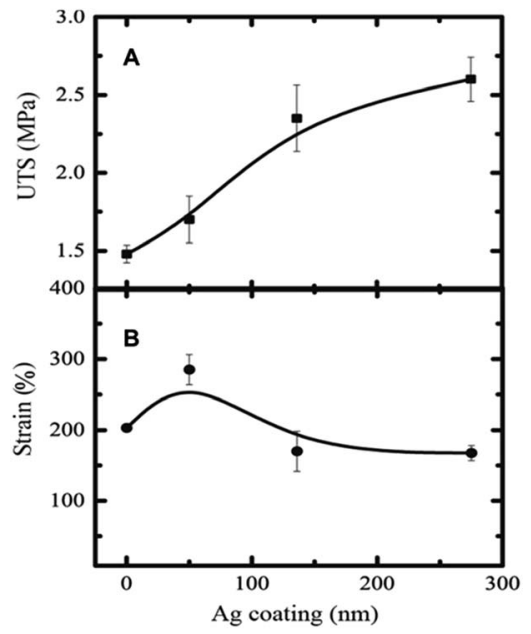

Fig. 4 (A) The ultimate tensile strength (UTS) of the Ag-coated fibrous scaffold also increased with Ag coating. (B) The tensile strain of the Agcoated scaffold fibers did not show substantial increase with Agcoating.

compared to the pristine fibrous scaffold due to more tightly packed fibers' morphology and higher diameter with a low pore size (Fig. 2). It could also be speculated that the reduction in pore size leads to more friction between the fibers, which leads to higher tensile strength. The morphology of the fibrous scaffold after mechanical testing is shown in Fig. 5. The Ag coating is slightly detached after a mechanical stretching of around 200-300\%. The pristine scaffold fibers showed twisting and stretching behaviour after the mechanical stretching whereas Ag-coated scaffold appeared to show less twisting and more stretching behaviour probably due to a strong Ag-fiber interaction where part of the strain was transferred to the coating (Fig. 5). Alternatively, the mechanical locking of the junctions by the Ag coating can potentially prevent easy slide of the fibers on each other.

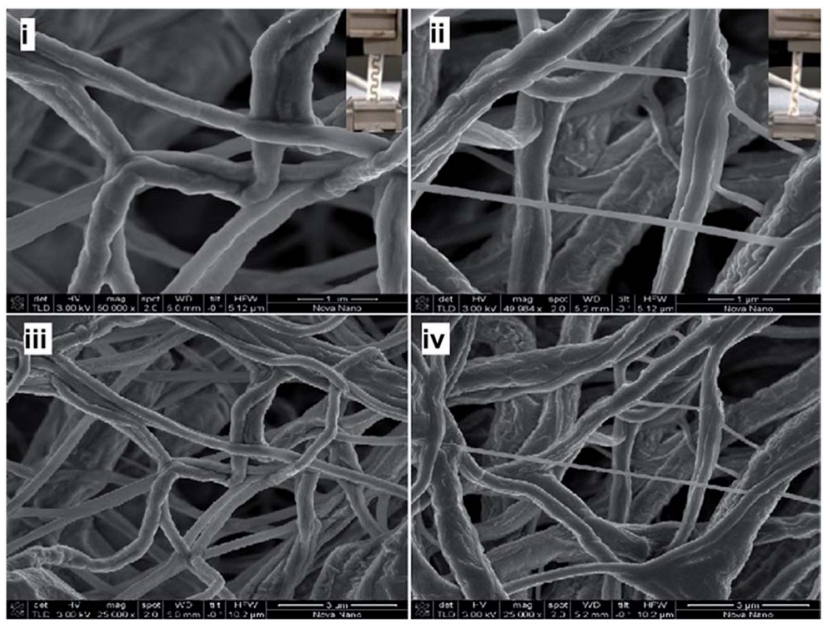

Fig. 5 SEM images of the pristine fibrous PGS/PCL scaffold and Agcoated scaffolds after mechanical testing: (i), (ii) are for pristine PGS/ $P C L$ and (iii), (iv) are for $\mathrm{Ag}$-coated fibrous composites (275 nm). 
The origin of mechanical enhancement of these fibrous scaffolds and Ag-coated scaffolds might also originate from an improved structure, which can be probed using thermal stability measurements using the differential scanning calorimetry (DSC) analysis and dynamic mechanical analysis (DMA). For the pristine scaffold, the DSC curve on heating showed a sharp melting peak ${ }^{45}$ at $58.16{ }^{\circ} \mathrm{C}$ (Fig. 6A). The melting temperature for $275 \mathrm{~nm}$ Ag-coated scaffolds was reduced to $56.55{ }^{\circ} \mathrm{C}$. The change in enthalpy (absorbed heat as the area under the DSC peak) was found to be $19.5 \mathrm{~J} \mathrm{~g}^{-1}$. The endothermic transition temperature $\left(T_{\mathrm{m}}\right)$ is decreased by about $2{ }^{\circ} \mathrm{C}$ at $275 \mathrm{~nm} \mathrm{Ag}$ coating on the fibrous scaffold (Fig. S3 $\dagger$ ), but the transition enthalpy remained constant at $19.5 \pm 0.5 \mathrm{~J} \mathrm{~g}^{-1}$ within experimental errors (Fig. S3 $\dagger$ ). The intensity of the endothermic peak slightly increased for the Ag-coated fibrous scaffolds, which might be due to the heat transfer between the fibers and the silver coating.

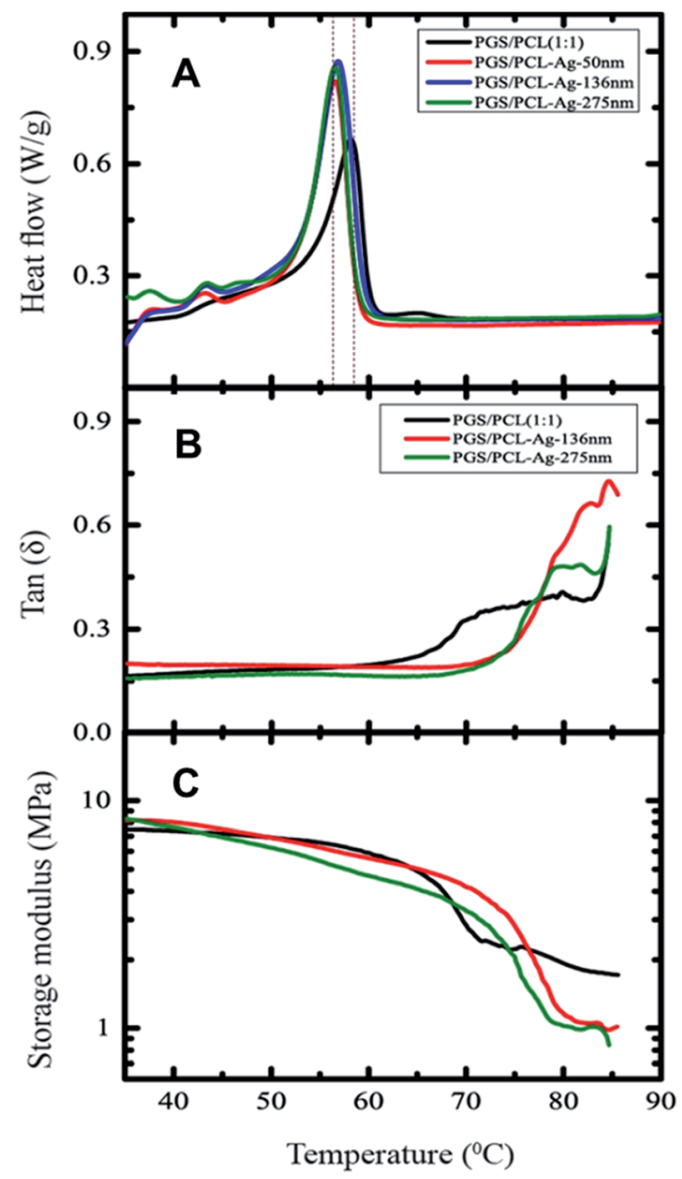

Fig. 6 Thermo-mechanical characteristics of the pristine fibrous scaffold of PGS/PCL and Ag-coated fibrous scaffold. (A) Differential scanning calorimetry measurements of pristine fibrous scaffold of PGS/PCL as well as Ag-coated coated ones. The coated scaffold showed enhancement of crystallization with increasing Ag coating. (B) damping ratio $(\tan \delta$ ) versus temperature which shows an enhancement in melting transition temperature of the fibrous scaffold. (C) The storage moduli versus temperature measurements showed that the Ag coating extended mechanical integrity of fibrous scaffold well beyond the fiber melting temperature.
We examined the melting transition temperature of fibrous and $\mathrm{Ag}$ coated scaffolds from the viscoelastic properties measured by DMA (Fig. 6B). The melting temperature of the pristine fibrous scaffolds decreased by almost 5 degrees, when Ag-coated. Similarly, the amplitude of the melting transition temperature also increased for Ag-coated composite scaffolds (Fig. 6A). Further, the storage modulus $\left(E^{\prime}\right)$ of Ag-coated composite fibrous scaffolds increased compared to the pristine scaffold (Fig. 6C) and this is probably due to the heat transfer between the fibers and the silver coating.

We also assessed the scaffolds' thermal stability using thermogravimetric analyses (TGA) shown in Fig. 7A. The mass-loss of fibers in Ag-coated fibrous scaffold is reduced when the thickness reaches $275 \mathrm{~nm}$. Furthermore, it also showed that the degradation temperature of the composites shifted to a higher temperature with the Ag coating (Fig. 7A). The thermal stability improved by $100 \mathrm{C}$ for Ag-coated $(275 \mathrm{~nm})$ fibrous scaffold however. The sample coated with a $130 \mathrm{~nm}$ film of $\mathrm{Ag}$ behaved like the pristine samples probably because the $\mathrm{Ag}$ coating evaporated too fast. The pristine and the one coated with a $136 \mathrm{~nm}$ film of $\mathrm{Ag}$ decomposed the same way. A slow mass loss observed until $400{ }^{\circ} \mathrm{C}$ and then a sheer drop.

The release of $\mathrm{Ag}$ ions or nanoparticles are a potential candidate for wound related antibacterial activity. ${ }^{33,34}$ The concentration of $\mathrm{Ag}$ ions released from the scaffold also needed

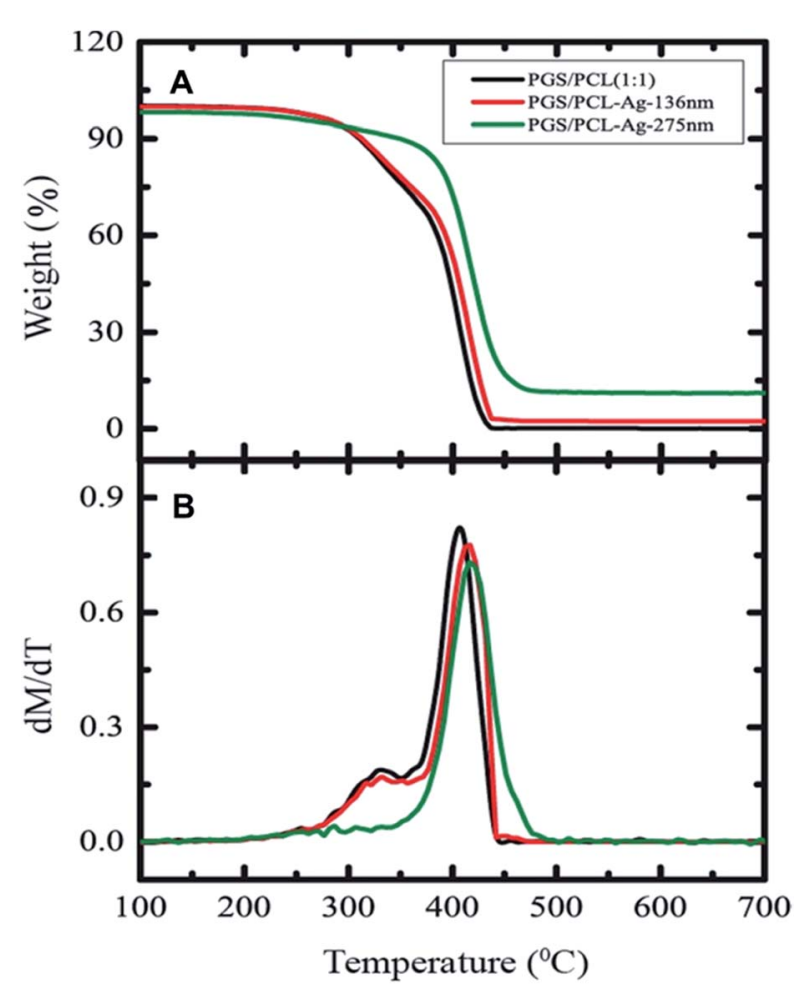

Fig. 7 Thermal stability of the pristine fibrous scaffold of PGS/PCL and Ag-coated fibrous scaffold. (A) Thermogravimetric analysis (TGA) of PGS/PCL scaffold, and Ag-coated fibers under a nitrogen atmosphere. The mass loss in fibers associated with burning fibrous scaffold off reduced in the presence of the Ag coating. (B) Examination of the rate of mass loss showed that decomposition of fibrous scaffold significantly shifted to higher temperature with the Ag coating. 


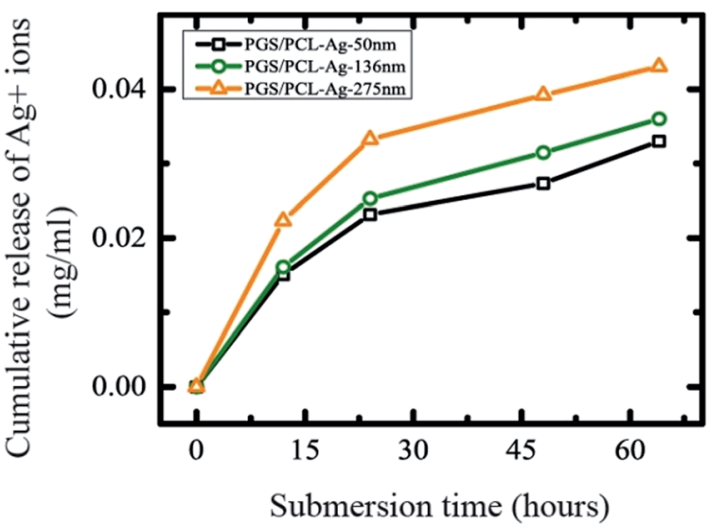

Fig. 8 Cumulative release of $\mathrm{Ag}^{+}$ion concentration of different $\mathrm{Ag}$ coated fibrous PGS/PCL scaffolds in DI water as a function of time using ICP-OES. The relation between the $\mathrm{Ag}$ ions release and the thickness is surprisingly non-linear.

to be identified. To investigate the potential antibacterial activity by controlled release of $\mathrm{Ag}^{+}$ions from fiber mats, we used total immersion method in DI water. We soaked Ag-coated
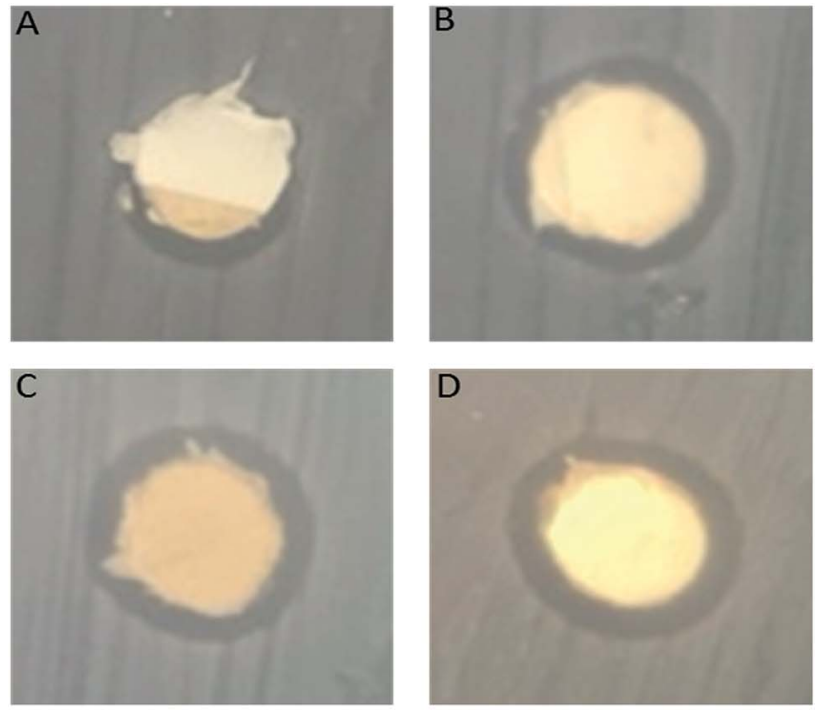

12 $E$

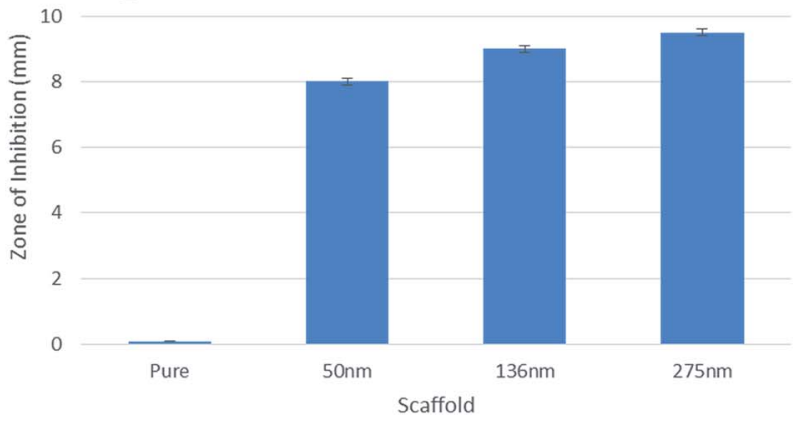

Fig. 9 Antimicrobial effect of loaded polymer against E. coli bacteria. In comparison with non-loaded side of material (A) zone of inhibition appears clearly around the material loaded (B-D). (E) The diameter of the zone of inhibition is reported in the table for a duplicate experiment $(n=2)$. This shows a clear antimicrobial effect of the coated composite. scaffold mats in DI water $(2 \mathrm{~mL})$ for several days and measured $\mathrm{Ag}^{+}$ions concentration by ICP-OES (Fig. 8). The release profile of silver ions followed similar trends for the different Ag-coated scaffolds (Fig. 8). The amount of silver released was about 5$10 \%$ even after 3 days. The slow and controlled release of $\mathrm{Ag}$ from the engineered scaffolds prevents any possible toxicity, while maintaining the antibacterial activity of the scaffolds. To address these potential biomedical applications, we tested the antibacterial effect of the Ag coatings on the PGS/PCL scaffold. We observed that the zone of inhibition against $E$. coli exists in all cases even with the minimal $50 \mathrm{~nm} \mathrm{Ag}$ coating (Fig. 9A-D). In addition, as the coating level was increased so did the zone of inhibition (Fig. 9E). Thus, the engineered scaffolds hold a great promise in many clinical applications such as surgical meshes, wound dressings, and antibacterial sutures.

\section{Discussion}

The $\mathrm{Ag}$ films can be deposited using various methods however $\mathrm{Ag}$ being a nobel metal sometimes exhibits poor adhesion to substrates. By utilizing a RF coating method we were successful in providing a uniform film on the surface of the fibers of the electrospun blend of PGS/PCL polymers (Fig. S1 $\dagger$ ). Through the adjustment of RF sputtering parameters such as argon gas flow, gas pressure, power intensity and deposition time we could generate a high enough kinetic energy for the sputtered $\mathrm{Ag}$ that can enhance their interaction with the polymer substrate surface leading to better adhesion without causing thermal damage and defect to the nanofibrous substrates. FTIR, revealed that the polymer characteristics were not entirely lost although they were greatly reduced (Fig. 1 and S2 $†$ ). This indicates that the film is thin enough that the chemical characteristics of the polymer can still be surface-active. The mechanical properties were enhanced greatly probably because the friction between the fibers was enhanced by the coating. We speculate that the reason for significant variation in the mechanical properties of the substrate by addition of such a thin coating might be due to interlocking of fibers and avoiding the slippage and increasing the friction between adjacent fibers on top of each other. This theory is well in agreement with our data showing a direct relationship between the ultimate stress as and the coating thickness without any significant variation in the ultimate strain. The mass loss of these fibers was improved when the thickness is beyond $200 \mathrm{~nm}$ probably because the heat transfer of the film improves the degradation of the film. When the $\mathrm{Ag}$ film is relatively thin, the film mass loss follows two regimes; a slow regime followed by a faster loss. This was probably due to the degradation of the two polymers that phase separate before they degrade totally. When the film is thicker, the mass loss occurs at a higher temperature-higher by $12{ }^{\circ} \mathrm{C}$ than the degradation temperature of the pristine polymer blend. The absence of the slow slope of the mass loss is probably due to the heat stored in the film and released into the fiber causing the whole polymer blend to degrade. We used this coating to test the possibility of using this polymer for potential controlled release systems and we found that the release of Ag ions is steady in time and does not commensurate with the 
nanofilm thickness. For a thickness that was $>4$ times thicker, the release was not $>4$ times higher, the release profile of the $\mathrm{Ag}$ ions from film is comparable between the samples. Antibacterial testing of the composite sheets showed promising results for biomedical applications such as surgical meshes or bandages. This could therefore provide a stepping stone to a controlled release system for possible antibacterial applications in wound dressings.

\section{Experimental}

\section{Materials}

The chemicals and solvents were purchased from SigmaAldrich. PGS pre polymer was synthesized based on the previously reported method. ${ }^{\mathbf{1 8 , 4 6}}$ Briefly, glycerol and sebacic acid with 1 : 1 molar ratio were placed at $120^{\circ} \mathrm{C}$ under high vacuum to make pre-polymer. Then the PGS and PCL blends were dissolved with $1: 1$ in anhydrous chloroform: ethanol (9:1) solution with oral polymer concentration of $20 \%(\mathrm{w} / \mathrm{v})$. To get a homogeneous mixture, the solutions were allowed to mix well overnight at room temperature prior to electrospinning process. Main paragraph text follows directly on here.

\section{Electrospinning}

The PGS and PCL were dissolved at the ratio of $1: 1$ in anhydrous chloroform : ethanol (9:1) mixture. An electrical field of $19.5 \mathrm{kV}$ over a distance of $15 \mathrm{~cm}$ was applied. The flow rate of the prepolymer composite was set to $1.5 \mathrm{~mL} \mathrm{~h}^{-1}$ and a $27 \mathrm{G}$ needle was used during electrospinning process. The electrospun sheet was then dried under the electrospinning system fan overnight at airflow of $12 \mathrm{~m}^{3} \mathrm{~h}^{-1}$ to remove any remaining solvent prior to further use.

\section{Scanning electron microscopy (SEM)}

The SEM images of the coated sheets were acquired using a JEOL JSM 7600F $(10 \mathrm{kV})$ to determine the structural features of the fabricated electrospun sheets. Dried electrospun samples were mounted on copper stubs using conductive carbon adhesive tape and used for SEM analysis.

\section{Fiber coatings}

Patterns were fabricated from Ag using a radio frequency (RF) magnetron sputtering (DC/RF Magnetron Sputter System, Syskey Technologies, Taiwan) technique. High purity target $(99.999 \%)$ of $\mathrm{Ag}(3 \times 0.6 \mathrm{inch})$ was used. To prepare nanocrystalline Ag thin films, the plasma was generated inside the chamber using argon gas with flow rate $20 \mathrm{sccm}$ at RF power of 100 watt while the base pressure and operating pressure, were adjusted at $1 \times 10^{-6}$ and $5 \times 10^{-3}$ Torr. The substrate rotation, target-substrate distance and deposition time were $15 \mathrm{rpm}$, $14 \mathrm{~cm}$ and $1000 \mathrm{~s}$ respectively. Thickness measurement of the samples were assessed using Dektak XT, Bruker, surface profiler with a stylus of 12.5 micrometer and stylus force $3 \mathrm{mg}$. The thickness of the film could be controlled by increasing or decreasing the deposition time.

\section{Mechanical characterization}

The tensile stress $(\sigma)$ was measured as a function of tensile strain $(\varepsilon)$ at a rate of $1 \mathrm{~mm} \mathrm{~s}^{-1}$ at room temperature with a $5 \mathrm{~N}$ load cell using DMA 800 system (TA Instruments). For the tensile measurements, we followed the ASTM standard ASTM D 882 including the testing of plastic sheets with the thickness less than $0.25 \mathrm{~mm}$.

\section{Thermal analyses}

Differential scanning calorimetry (DSC) measurements were carried out with a DSC-204 F1 (Netzsch) at a heating rate of $50{ }^{\circ} \mathrm{C} \mathrm{min}^{-1}$. Thermogravimetric analysis (TGA) was carried out under atmospheric $\mathrm{N} 2$ over a temperature range of 25 to $800{ }^{\circ} \mathrm{C}$ using a TG-209F (Netzsch) at a heating rate of $5{ }^{\circ} \mathrm{C} \mathrm{min}^{-1}$.

\section{Fourier transforms infrared spectroscopy (FTIR)}

The chemical compositions of composite fibrous scaffold were collected by using a Fourier transform infrared spectroscopy (FTIR-6700 Smart FTIR spectrometer ranging from $4000 \mathrm{~cm}^{-1}$ to $\left.400 \mathrm{~cm}^{-1}\right)$.

\section{Inductively coupled plasma optical emission spectrometry (ICP-OES)}

The cumulative $\mathrm{Ag}^{+}$ions activity in deionized water (DI) was calculated by a Varian inductively coupled plasma optical emission spectrometer (ICP-OES).

\section{Antibacterial testing}

To test the antimicrobial effect of the scaffolds, one isolated colony of the Top $10 \mathrm{E}$. coli strain (Invitrogen) transformed with pUC18 plasmid giving an ampicillin resistance was put in culture in LB media containing ampicillin $\left(100 \mu \mathrm{g} \mathrm{mL} \mathrm{m}^{-1}\right)$ at $37{ }^{\circ} \mathrm{C}$ overnight. $100 \mu \mathrm{L}$ of this culture were spread on the surface of LB-agar plates containing ampicillin $\left(100 \mu \mathrm{g} \mathrm{mL} \mathrm{m}^{-1}\right)$ to get a mat of bacteria. Disks of non-loaded polymer and loaded polymer $(50,136$ and $275 \mathrm{~nm})$ were placed on the surface of the plates in duplicates and incubated at $37{ }^{\circ} \mathrm{C}$ overnight.

\section{Conclusions}

We used a RF-assisted technique to coat a nanofilm of silver on electrospun scaffolds of PGS/PCL for controlled release of Ag to render them antibacterial for biomedical applications. We found that the coated scaffolds were stable and possessed suitable mechanical properties. The Ag coating was both thermally and mechanically strong and the Ag ions release were gradual and controlled. These characteristics minimize the safety concerns regarding the potential toxicity of these constructs. Pathogenic antibacterial testing revealed that these composites are good candidates for biomedical applications such as bandages and wound dressings. The sheets produced in this study have superior mechanical and thermal properties as well promising biomedical applications. 


\section{Acknowledgements}

This project was funded by the National Plan for Science, Technology and Innovation (MAARIFAH) - King Abdulaziz City for Science and Technology - the Kingdom of Saudi Arabia award number (12-MED3096-3). The authors thank the Science and Technology Unit, King Abdulaziz University. PK and SC thank KAUST for its support.

\section{References}

1 C. K. Sen, G. M. Gordillo, S. Roy, R. Kirsner, L. Lambert, T. K. Hunt, F. Gottrup, G. C. Gurtner and M. T. Longaker, Wound Repair Regen., 2009, 17, 763-771.

2 B. S. Atiyeh, M. Costagliola, S. N. Hayek and S. A. Dibo, Burns, 2007, 33, 139-148.

3 V. R. Driver, M. Fabbi, L. A. Lavery and G. Gibbons, J. Vasc. Surg., 2010, 52, 17S-22S.

4 A. B. Association, American Burn Association, Chicago, 2011.

5 L. K. Branski, G. G. Gauglitz, D. N. Herndon and M. G. Jeschke, Burns, 2009, 35, 171-180.

6 F. H. Epstein, A. J. Singer and R. A. Clark, N. Engl. J. Med., 1999, 341, 738-746.

7 Y. Bayram, M. Parlak, C. Aypak and I. Bayram, Int. J. Med. Sci., 2013, 10, 19.

8 P. Kanmani and J. W. Rhim, Food Chem., 2014, 148, 162-169. 9 L. L. Zhang, Y. H. Jiang, Y. L. Ding, N. Daskalakis, L. Jeuken, M. Povey, A. J. O'Neill and D. W. York, J. Nanopart. Res., 2010, 12, 1625-1636.

10 Y. A. Arfat, S. Benjakul, T. Prodpran, P. Sumpavapol and P. Songtipya, Food Hydrocolloids, 2014, 41, 265-273.

11 Y. Hemar, L. H. Liu, N. Meunier and B. W. Woonton, Innovative Food Sci. Emerging Technol., 2010, 11, 432-440.

12 M. T. Sheu, J. C. Huang, G. C. Yeh and H. O. Ho, Biomaterials, 2001, 22, 1713-1719.

13 H. J. Kong, E. Wong and D. J. Mooney, Macromolecules, 2003, 36, 4582-4588.

14 A. Khademhosseini, J. P. Vacanti and R. Langer, Sci. Am., 2009, 300, 64-71.

15 M. Radisic, H. Park, T. P. Martens, J. E. Salazar-Lazaro, W. Geng, Y. Wang, R. Langer, L. E. Freed and G. VunjakNovakovic, J. Biomed. Mater. Res., 2008, 86, 713-724.

16 S. Redenti, W. L. Neeley, S. Rompani, S. Saigal, J. Yang, H. Klassen, R. Langer and M. J. Young, Biomaterials, 2009, 30, 3405-3414.

17 N. Masoumi, B. L. Larson, N. Annabi, M. Kharaziha, B. Zamanian, K. S. Shapero, A. T. Cubberley, G. CamciUnal, K. B. Manning, J. E. Mayer Jr and A. Khademhosseini, Adv. Healthcare Mater., 2014, 3, 929939.

18 Y. Wang, G. A. Ameer, B. J. Sheppard and R. Langer, Nat. Biotechnol., 2002, 20, 602-606.

19 Y. D. Wang, Y. M. Kim and R. Langer, J. Biomed. Mater. Res., Part A, 2003, 66, 192-197.

20 C. Fidkowski, M. R. Kaazempur-Mofrad, J. Borenstein, J. P. Vacanti, R. Langer and Y. Wang, Tissue Eng., 2005, 11, 302-309.
21 C. A. Sundback, J. Y. Shyu, Y. D. Wang, W. C. Faquin, R. S. Langer, J. P. Vacanti and T. A. Hadlock, Biomaterials, 2005, 26, 5454-5464.

22 A. Khademhosseini, R. Langer, J. Borenstein and J. P. Vacanti, Proc. Natl. Acad. Sci. U. S. A., 2006, 103, 24802487.

23 D. Motlagh, J. Yang, K. Y. Lui, A. R. Webb and G. A. Ameer, Biomaterials, 2006, 27, 4315-4324.

24 S. A. Catledge, W. C. Clem, N. Shrikishen, S. Chowdhury, A. V. Stanishevsky, M. Koopman and Y. K. Vohra, Biomed. Mater., 2007, 2, 142-150.

25 G. C. Engelmayr, M. Y. Cheng, C. J. Bettinger, J. T. Borenstein, R. Langer and L. E. Freed, Nat. Mater., 2008, 7, 1003-1010.

26 J. Gao, P. Crapo, R. Nerern and Y. D. Wang, J. Biomed. Mater. Res., Part A, 2008, 85, 1120-1128.

27 E. D. Boland, K. J. Pawlowski, C. P. Barnes, D. G. Simpson, G. E. Wnek and G. L. Bowlin, ACS Symp. Ser., 2006, 918, 188-204.

28 B. M. Baker, A. O. Gee, R. B. Metter, A. S. Nathan, R. A. Marklein, J. A. Burdick and R. L. Mauck, Biomaterials, 2008, 29, 2348-2358.

29 A. Balguid, M. P. Rubbens, A. Mol, R. A. Bank, A. J. Bogers, J. P. van Kats, B. A. de Mol, F. P. Baaijens and C. V. Bouten, Tissue Eng., 2007, 13, 1501-1511.

30 G. H. Kim, Biomed. Mater., 2008, 3, 025010.

31 C. Li, C. Vepari, H. J. Jin, H. J. Kim and D. L. Kaplan, Biomaterials, 2006, 27, 3115-3124.

32 A. Balguid, A. Mol, M. H. van Marion, R. A. Bank, C. V. Bouten and F. P. Baaijens, Tissue Eng., Part A, 2009, 15, 437-444.

33 F. N. Almajhdi, H. Fouad, K. A. Khalil, H. M. Awad, S. H. Mohamed, T. Elsarnagawy, A. M. Albarrag, F. F. AlJassir and H. S. Abdo, J. Mater. Sci.: Mater. Med., 2014, 25, 1045-1053.

34 N. Chaubey, A. K. Sahoo, A. Chattopadhyay and S. S. Ghosh, Biomater. Sci., 2014, 2, 1080-1089.

35 P. S. Gungor-Ozkerim, T. Balkan, G. T. Kose, A. S. Sarac and F. N. Kok, J. Biomed. Mater. Res., Part A, 2014, 102, 18971908.

36 J. Jiang, J. Xie, B. Ma, D. E. Bartlett, A. Xu and C. H. Wang, Acta Biomater., 2014, 10, 1324-1332.

37 H. Wang, Y. Li, S. Jiang, P. Zhang, S. Min and S. Jiang, J. Appl. Polym. Sci., 2014, 131, 40903.

38 Y. Y. Tseng and S. J. Liu, Nanomedicine, 2015, 10, 17851800.

39 S. Sant, D. Iyer, A. K. Gaharwar, A. Patel and A. Khademhosseini, Acta Biomater., 2013, 9, 5963-5973.

40 J. L. Ifkovits, J. J. Devlin, G. Eng, T. P. Martens, G. VunjakNovakovic and J. A. Burdick, ACS Appl. Mater. Interfaces, 2009, 1, 1878-1886.

41 J. L. Ifkovits, R. F. Padera and J. A. Burdick, Biomed. Mater., 2008, 3, 034104.

42 S. Sant, C. M. Hwang, S. H. Lee and A. Khademhosseini, J. Tissue Eng. Regener. Med., 2011, 5, 283-291.

43 W. Cai and L. L. Liu, Mater. Lett., 2008, 62, 2171-2173. 
44 T. Elzein, M. Nasser-Eddine, C. Delaite, S. Bistac and P. Dumas, J. Colloid Interface Sci., 2004, 273, 381-387.

45 S. Salehi, T. Bahners, J. S. Gutmann, S. L. Gao, E. Mader and T. A. Fuchsluger, RSC Adv., 2014, 4, 16951-16957.
46 N. Masoumi, A. Jean, J. T. Zugates, K. L. Johnson and G. C. Engelmayr Jr, J. Biomed. Mater. Res., Part A, 2013, 101, 104-114. 